\title{
Transrectal Prostate Biopsy and Fiducial Marker Placement in a Standard 1.5T Magnetic Resonance Imaging Scanner
}

\section{Robert C. Susil,*, $\dagger$ Cynthia Ménard,* Axel Krieger, Jonathan A. Coleman, Kevin Camphausen,}

Peter Choyke, Gabor Fichtinger, Louis L. Whitcomb, C. Norman Coleman and Ergin Atalar

From the Departments of Biomedical Engineering (RCS, EA), Radiology (AK, EA), Computer Science (GF) and Mechanical Engineering (LLW), Johns Hopkins University School of Medicine, Baltimore, Maryland, the Radiation Oncology Branch (CM, KC, CNC) and Urologic Oncology Branch (JAC), National Cancer Institute, and Department of Radiology, Clinical Center (PC), National Institutes of Health, DHHS, Bethesda, Maryland, the Department of Electrical and Electronics Engineering (EA), Bilkent University, Ankara, Turkey, and University of Toronto, Princess Margaret Hospital, Toronto, Canada (CM)

Purpose: We investigated the accuracy and feasibility of a system that provides transrectal needle access to the prostate concurrent with 1.5 Tesla MRI which previously has not been possible.

Materials and Methods: In 5 patients with previously diagnosed prostate cancer, MRI guided intraprostatic placement of gold fiducial markers (4 procedures) and/or prostate biopsy (3 procedures) was performed using local anesthesia.

Results: Mean procedure duration was 76 minutes and all patients tolerated the intervention well. Procedure related adverse events included self-limited hematuria and hematochezia following 3 of 8 procedures (all resolved in less than 1 week). Mean needle placement accuracy was $1.9 \mathrm{~mm}$ for the fiducial marker placement studies and $1.8 \mathrm{~mm}$ for the biopsy procedures. Mean fiducial marker placement accuracy was $4.8 \mathrm{~mm}$ and the mean fiducial marker placement accuracy transverse to the needle direction was $2.6 \mathrm{~mm}$. All patients who underwent the procedure were able to complete their course of radiotherapy without delay or complication.

Conclusions: While studies of clinical usefulness are warranted, transrectal $1.5 \mathrm{~T}$ MRI guided prostate biopsy and fiducial marker placement is feasible using this system, providing new opportunities for image guided diagnostic and therapeutic prostate interventions.

Key Words: magnetic resonance imaging; prostate; prostatic neoplasms; biopsy; radiology; interventional

$\mathrm{M}$ agnetic resonance imaging with an endorectal coil has a potentially valuable role in the diagnosis of prostate cancer. While the excellent soft tissue contrast and functional imaging capabilities of MRI provide high sensitivity for detecting prostate tumors, ${ }^{1}$ the low specificity of MRI has limited its role in prostate cancer diagnosis and staging. ${ }^{2}$ Ideally, an MRI platform that provides for image guided prostate biopsy could maintain the sensitivity of MRI while gaining the specificity of tissue biopsy.

Previously, prostate biopsy and brachytherapy procedures have been performed using low field strength (eg 0.2 or $0.5 \mathrm{~T}$ ) open scanner architectures. ${ }^{3,4}$ While these systems provide easier access to the patient, they do not produce the highest quality MR images, have limited potential for functional and spectroscopic imaging, and are less widely available. To improve image quality, some groups have investigated hybrid approaches in which previously ac-

Submitted for publication September 20, 2004

Study received institutional review board approval.

* Equal study contribution.

$\dagger$ Supported by an NIH training grant.

Supported by United States Army Prostate Cancer Research Program Award DAMD17-01-1-0064, NSF ERC 9731478 and an NSF ERC PER grant.

$\ddagger$ Correspondence: Johns Hopkins University, Traylor BuildingRoom 330, 720 Rutland Ave., Baltimore, Maryland 21205 (telephone: 410-955-9617; FAX: 443-287-6730; e-mail: eatalar@jhu.edu).

See Editorial on page 16. quired 1.5T MR images are registered to images acquired with a lower field strength scanner, where the actual intervention is performed. ${ }^{5,6}$ Others have registered previously acquired $1.5 \mathrm{~T} \mathrm{MR}$ images with intraoperative ultrasound images., ${ }^{7,8}$ While both of these image registration approaches simplify the intervention itself, registration between image sets, particularly in highly deformable organs such as the prostate, can introduce inaccuracies.

There are currently no systems that allow for transrectal needle placement concurrent with the acquisition of diagnostic quality MR images in a closed 1.5T MR scanner architecture. An obvious application of such a system is MR guided diagnostic prostate biopsy. However, performing MRI guided minimally invasive therapeutic interventions is also of interest. ${ }^{9}$ In the research setting, such a system could provide for direct histological validation and molecular analysis of various prostate MR imaging techniques.

In this technical pilot study, we investigate the feasibility and tissue targeting accuracy of a novel system that allows for transrectal needle access to the prostate while a patient is imaged inside of a closed 1.5T MRI scanner. In 5 patients with previously diagnosed localized prostate cancer, this device was used for 4 gold fiducial marker placement procedures and 3 MRI guided prostate biopsy procedures. Although not discussed, the fiducial markers were used to guide external beam radiation therapy and the tissue biopsies, collected from normal and cancerous prostate tissue, were used to study the molecular effects of radiation ther- 
apy. As this is an initial investigation of clinical feasibility, we do not attempt to prove a clinical role for this device, but rather, only to study its feasibility and targeting accuracy.

\section{MATERIALS AND METHODS}

Interventional device. A custom made MR compatible device was designed for the interventional procedures. ${ }^{9,10}$ A 23 mm-diameter hollow endorectal sheath, placed in the rectum at the beginning of the procedure, remains immobile throughout the intervention (fig. 1, A). This sheath includes an integrated $20 \mathrm{~mm}$ diameter single turn imaging coil surrounding an anterior window that allows for needle access to the prostate. An $18 \mathrm{~mm}$ diameter cylindrical needle guide fits inside the stationary rectal sheath, contains MR tracking microcoils (allowing for device registration), and has a curved needle channel (allowing the needle, inserted axially, to exit at a 36-degree angle, passing through the rectal wall and into the prostate, fig. $1, B$ ). The cylindrical needle guide is mounted on a positioning stage (fig. 1, C) which contains the mechanism that converts the rotation of 2 flexible control rods (S.S. White Technologies Inc., Piscataway, New Jersey, fig. 1, D), each extending to the edge of the scanner bore, into rotation and translation of the needle guide. Finally, the positioning stage is attached to an immobilization arm mounted on a linear rail (fig. $1, D$ ). Unless otherwise noted, all parts of the device were fabricated from Ultam plastic (GE Plastics, Brea, California).

Because the device has a coaxial design, the central axis provides an open channel for needle passage. The needles are inserted from the back of the device, pass through its central axis, are curved within the needle guide, and emerge from the lateral wall of the needle guide. The $18 \mathrm{G}$ marker placement needles were custom fabricated from nitinol (MRI-Devices Daum, Schwerin, Germany). To accommodate standard 14 gauge spring loaded MRI compatible biopsy guns (MRI-Devices Daum), a straight needle path was needed. Therefore, for the biopsy procedures, a needle guide with 20 and 30-degree straight needle channels and a modified endorectal sheath were used (fig. 2).

Patient enrollment and preparation. After providing informed consent, 5 patients were enrolled in an investigational protocol reviewed and approved by our institutional review board. All patients were previously diagnosed with clinically localized prostate cancer (via TRUS guided biopsy) and were scheduled to receive a standard course of conformal external beam radiation therapy. Four of the patients underwent MRI guided intraprostatic placement of 4 gold fiducial markers ( $4 \mathrm{~mm}$ long, $0.8 \mathrm{~mm}$ diameter, $24 \mathrm{~K}$ gold, Northwest Medical Physics Equipment, Lynnwood, Washington). The fiducial markers were subsequently used for daily assessment and adjustment of external radiation beam targeting. Three of the patients provided consent for MRI guided prostate biopsy. Because patients were already diagnosed with prostate cancer, the biopsy cores (collected from normal and cancerous prostate tissue) were used in an ongoing study of the molecular effects of radiation therapy.

Two days before and on the morning of the procedure, patients received prophylactic antibiotics (Levofloxacin, 500 mg po 4 times daily). Because this study was designed to

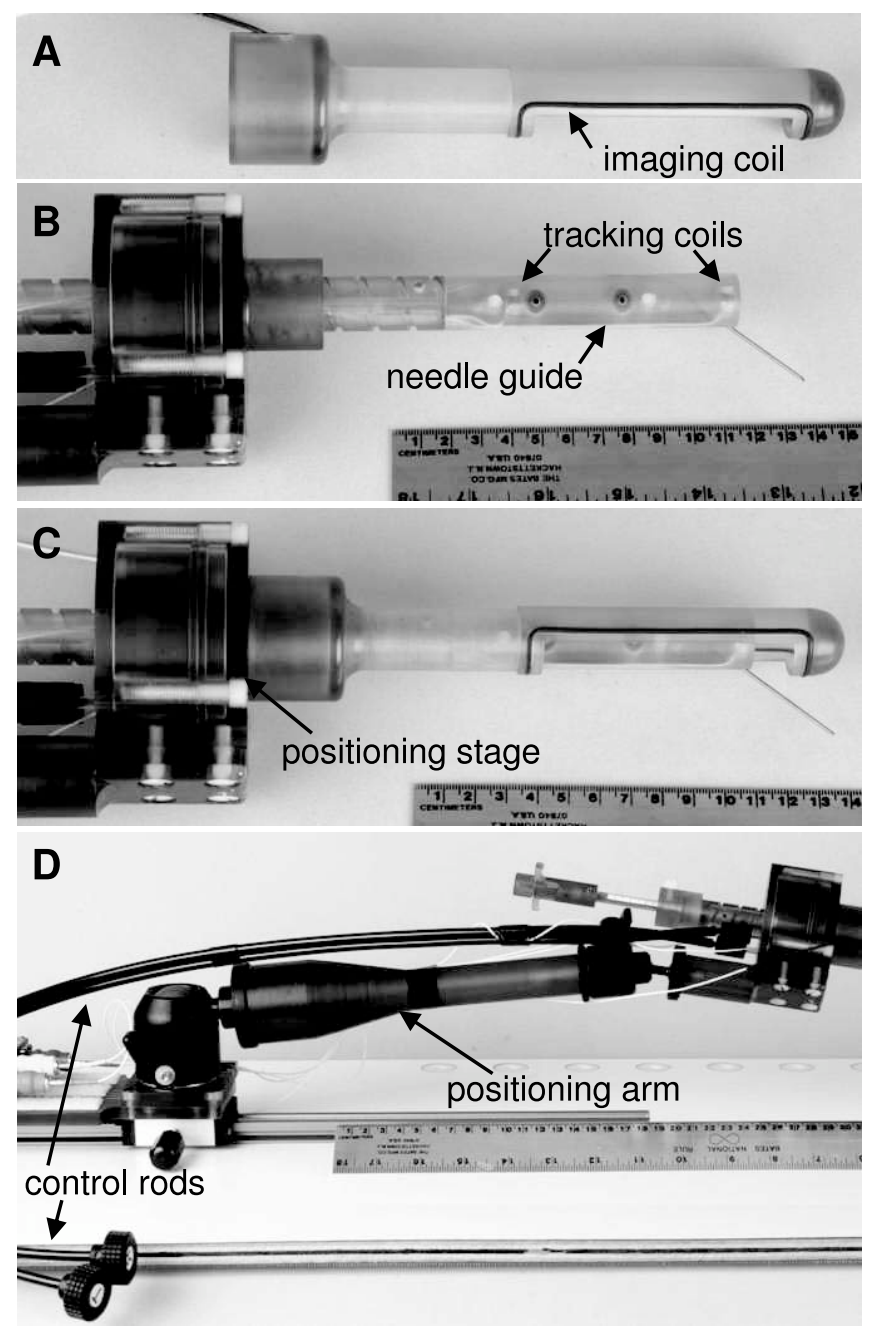

Fig. 1. Interventional system. $A$, stationary endorectal sheath with integrated $20 \mathrm{~mm}$ diameter single turn imaging coil minimizes tissue deformation during interventional procedure. $B$, cylindrical needle guide contains MR tracking microcoils and curved needle channel. $C$, positioning stage houses mechanism that converts rotation of 2 control rods into rotation and translation of needle guide, which fits inside endorectal sheath. $D$, positioning arm with 2 lockable aluminum ball joints and linear rail allows for easy device positioning and, subsequently, rigid immobilization.

investigate the accuracy of MRI guided needle placement, local anesthesia was administered via ultrasound guided transrectal injection of $10 \mathrm{ml}$ of $0.25 \%$ Marcaine along the periprostatic neurovascular bundles bilaterally immediately before the MR procedure. After the patient was positioned prone on the scanner table, the endorectal sheath was placed, the positioning stage was docked with the sheath, and then the entire system was immobilized (via the 2 ball joints and the linear rail) to prevent motion of the setup. The patients were advanced into the scanner where scout images were collected to verify the positioning of the device relative to patient anatomy (typically, zero or 1 positional adjustment was necessary to achieve the proper sheath depth).

Fiducial marker placement procedure. All interventions were performed on a GE Signa Excite 1.5 T MR scanner (GE Medical Systems). Four pulse sequences were used 

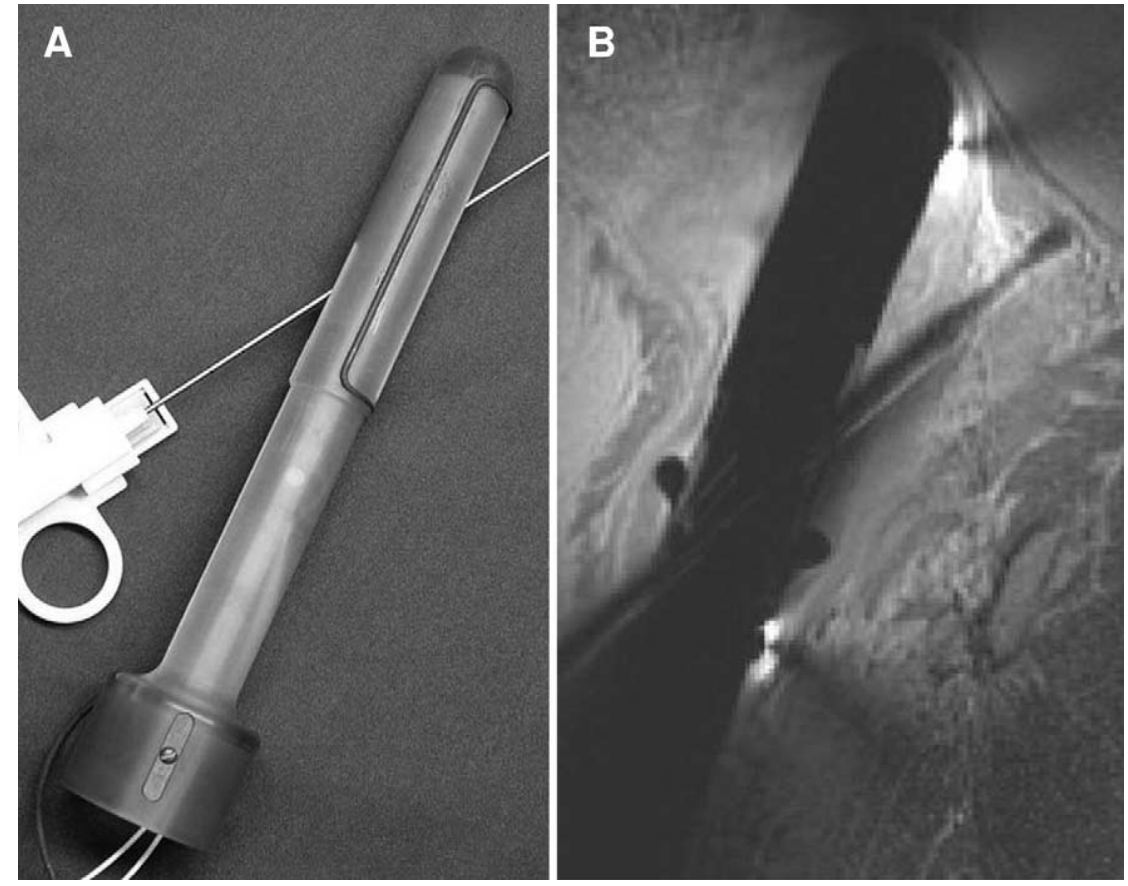

Fig. 2. Prostate biopsy needle guide and sheath. $A$, to accommodate 14 gauge spring-loaded biopsy guns, modified needle guide with 2 , straight needle channels (30 degrees and 20 degrees) and modified endorectal sheath were used. $B$, sagittal T1-weighted fast spin-echo image was acquired with biopsy needle in place. Using 1 of 2 needle channels, all sites within prostate could be accessed.

during these studies, namely 3 for anatomical imaging and a fourth for device tracking. After the patients were positioned in the scanner and scout images acquired, T2weighted fast spin-echo images were acquired in the axial and sagittal planes for visualization of the intraprostatic and periprostatic anatomy (FSE-XL, TE $102 \mathrm{msec}$, TR 6.0 seconds, BW $\pm 20.83 \mathrm{KHz}$, ETL 16, FOV $16 \mathrm{~cm}$, slice thickness $3 \mathrm{~mm}$, interslice spacing $0 \mathrm{~mm}, 256 \times 192$, NEX 3 , scan time 3:36). After acquisition, these images were automatically loaded into the visualization and targeting program, which was displayed on a screen (Da-Mat, Da-Lite Inc., Warsaw, IN) in the scanner room using an LCD projector (LP340b LCD Projector, Infocus Corp., Wilsonville, Oregon, fig. 3, B). A target for needle placement was selected and graphically marked on these images (fig. 4).

Next, a device tracking pulse sequence was run to determine the current position of the endorectal needle guide (via localization of the 3 tracking microcoils inside of the device). ${ }^{9}$ This 60 msec pulse sequence consists of 12 , dodecahedrally spaced 1D gradient echo projections (no slice selection is used, TE $2.3 \mathrm{msec}$, TR $5.0 \mathrm{msec}, \mathrm{BW} \pm 64 \mathrm{KHz}$, FA $1^{\circ}$, FOV $40 \mathrm{~cm}, 256$ readout points). By knowing the position of the target, the position of the needle guide and the needle path relative to the needle guide (determined via a prior device calibration), the amount of rotation and translation necessary to place the target on the needle trajectory could be calculated by the targeting program and displayed for the operator (fig. 4). The depth of needle insertion necessary to reach the target was also calculated and displayed as part of the user interface.

As the operator moved the needle guide via the 2 control knobs (fig. $3, A$ ), the tracking pulse sequence was run continuously and the remaining rotation and translation required were displayed. When the needle guide was correctly positioned, the tracking pulse sequence was stopped and the patient table withdrawn from the scanner to allow for needle insertion under direct supervision. To control the depth of needle insertion, a needle stop was set, placed at the back of the device, and the nitinol needle and cannula were introduced together. Because of the impact of deformation on tissue targeting accuracy, needles in the last 2 procedures were inserted $5 \mathrm{~mm}$ past the target site and then withdrawn back to the intended target depth before depositing the fiducial marker. As deformation was most pronounced at the base of the prostate, needles targeted in this area were inserted $8 \mathrm{~mm}$ past the target and then withdrawn back to the prescribed depth.

Needle position was confirmed using a T1-weighted fast spin-echo acquisition (FSE-XL, TE $9.5 \mathrm{msec}$, TR $1.25 \mathrm{sec}-$ onds, BW $\pm 31.25 \mathrm{KHz}$, ETL 4, FOV $16 \mathrm{~cm}$, slice thickness 3 $\mathrm{mm}$, interslice spacing $0 \mathrm{~mm}, 256 \times 256$, NEX 1 , scan time 1:20). A fiducial marker was then placed using the nitinol stylus and a cannula withdrawal technique, as described previously, ${ }^{9}$ and the prostate was imaged using a $\mathrm{T}^{*}$ weighted gradient-echo pulse sequence (GE, TE $15 \mathrm{msec}$, TR $467 \mathrm{msec}, \mathrm{BW} \pm 15.6 \mathrm{KHz}, \mathrm{FA} 30$ degrees, FOV $16 \mathrm{~cm}$, slice thickness $3 \mathrm{~mm}$, interslice spacing $0 \mathrm{~mm}, 256 \times 256$, NEX 1 , scan time 2:00). Because pure gold fiducial markers produce little susceptibility artifact and therefore little signal void, ${ }^{11}$ it was necessary to use $\mathrm{T} 2 *$-weighting to robustly visualize the fiducial markers. Following imaging of the fiducial marker, another target was selected (using the T2-weighted fast spin-echo images), and the needle/marker placement procedure repeated.

After placement of the 4 gold markers, patients were removed from the scanner suite and observed in the hospital until they successfully voided without difficulty. One week following the procedure, all patients were interviewed 

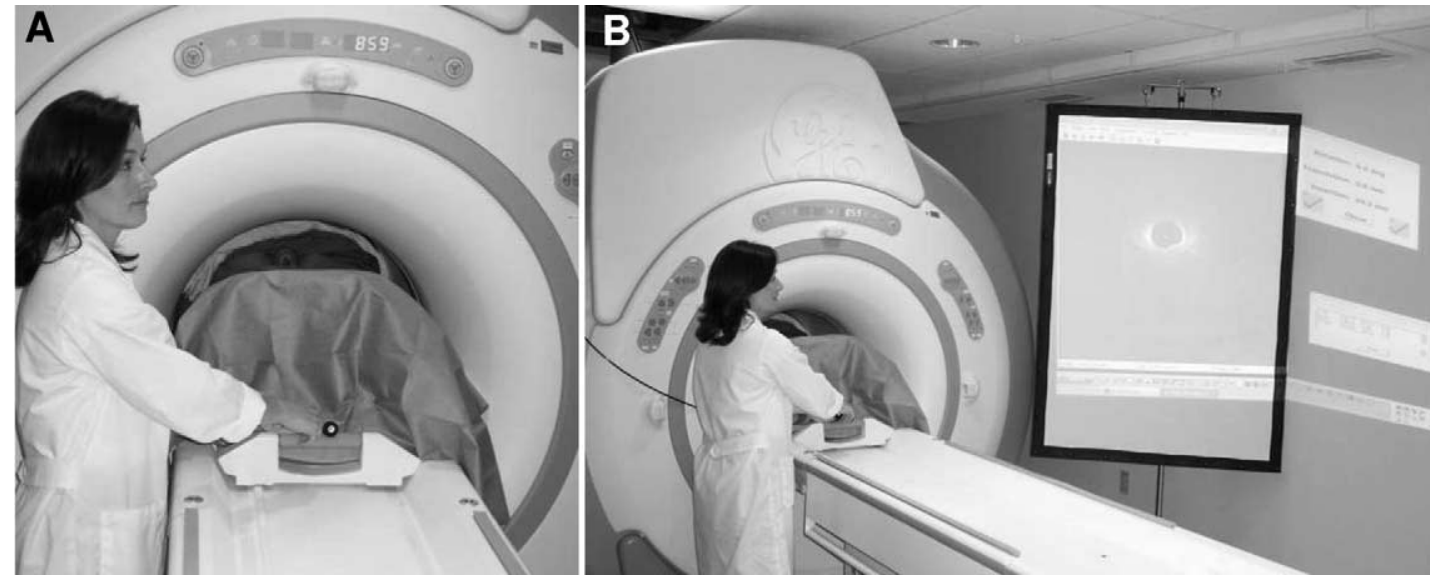

FIG. 3. MR scanner room setup. A, flexible control rods (2) extend to edge of scanner bore, allowing for rotation and translation of needle guide while prostate is located at scanner isocenter. $B$, image display and targeting application is projected onto screen in scanner room.

concerning potential adverse events (including hematuria, hematochezia, urinary retention, infection, and rectal discomfort).

Biopsy procedure. Patient preparation and scan room setup for the 3 MRI guided prostate biopsy procedures was identical to that described for the fiducial marker procedures. Targets were selected using axial T2-weighted fast spin-echo images and needle location was confirmed using axial T1-weighted fast spin-echo images (as described). Five $14 \mathrm{G}$ needle core biopsies were performed in each of 3 procedures.

\section{RESULTS}

Five patients were enrolled in the study, all of whom completed the protocol. With these patients, 4 fiducial marker

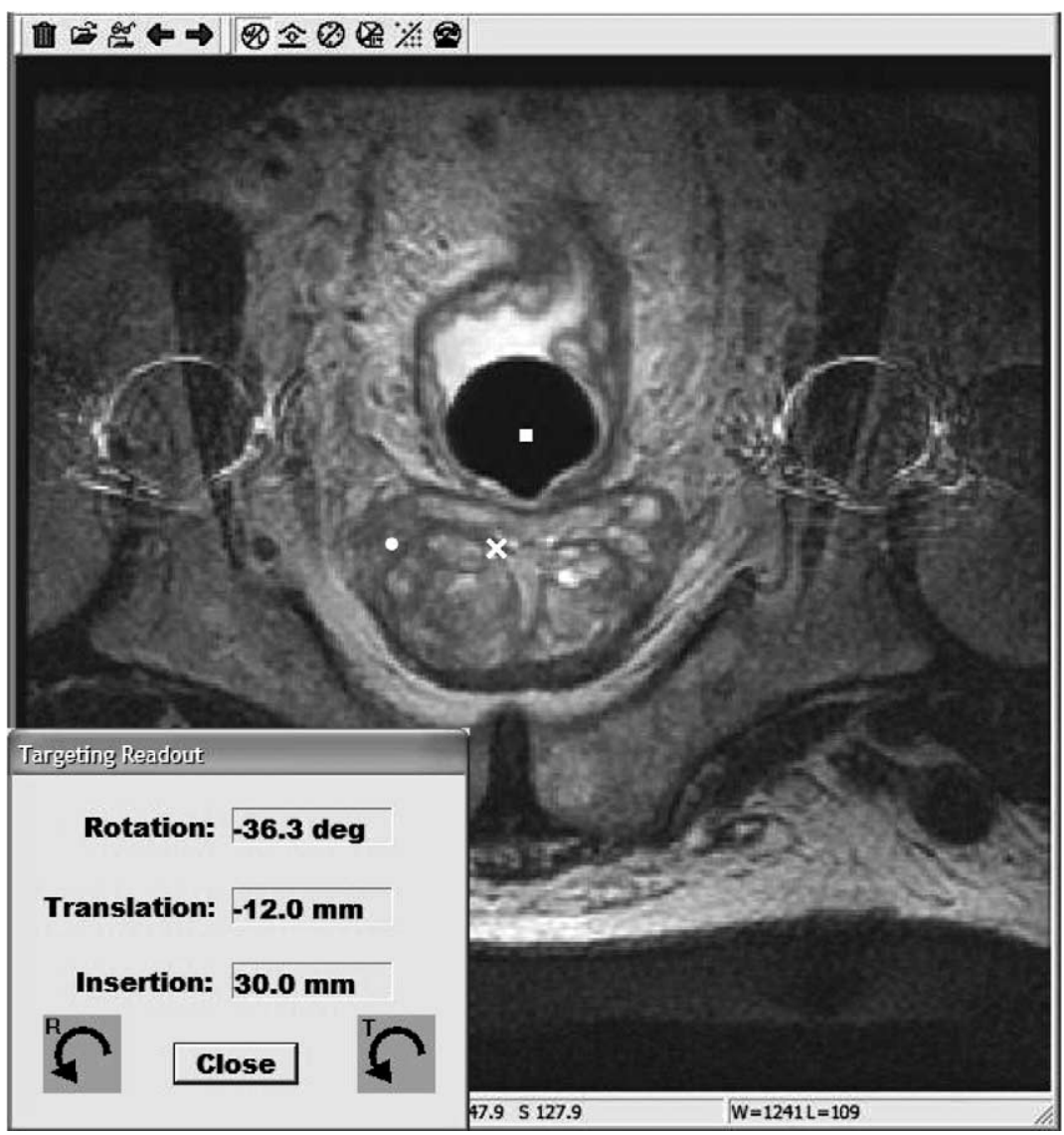

FIG. 4. Image display and targeting application. Target (white circle) is graphically selected within diffuse, low intensity region (corresponding to region cancerous tissue) in left posterior lobe of prostate. Intersection of image plane and projected needle path (white cross) as well as axis of needle guide (white square) are also shown. As device is moved, targeting commands and projected needle path are updated in real time. 
Target location

A

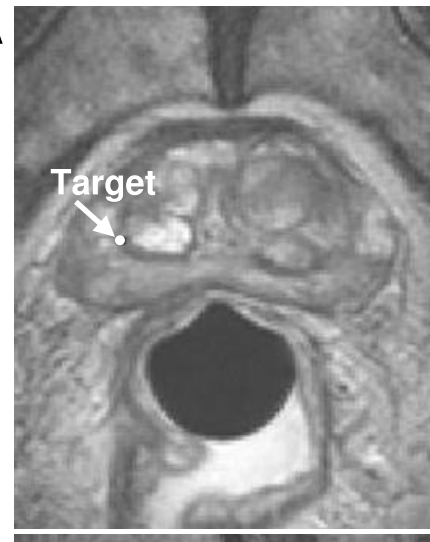

B

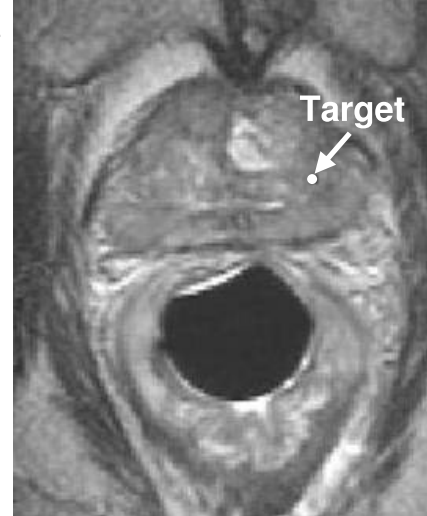

Needle inserted
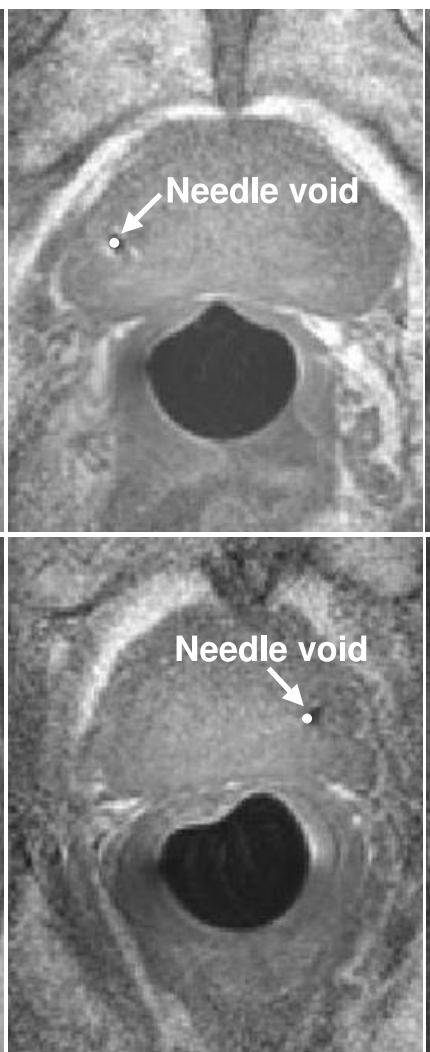

Marker deposited

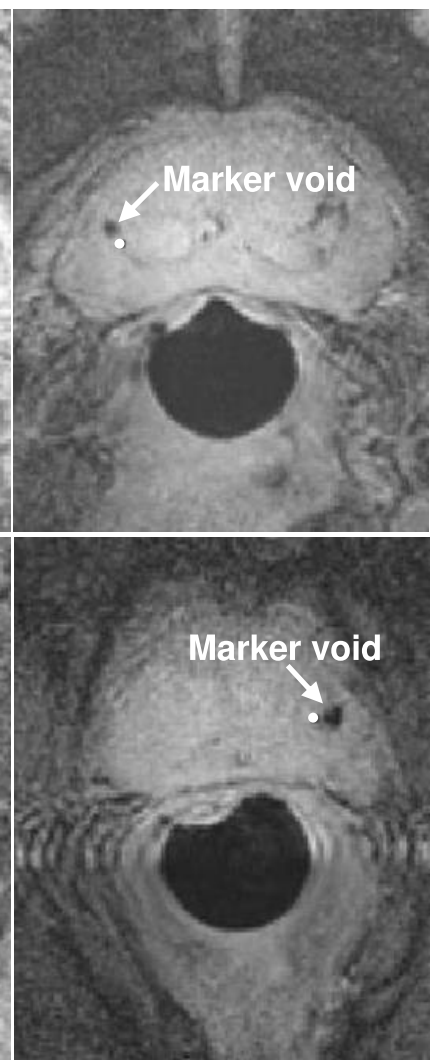

FIG. 5. Targeting, needle and fiducial-marker visualization images. Images from 2 patients ( $A$ and $B$, respectively), show images acquired during fiducial-marker placement procedure. Column 1, targets are selected on axial, T2-weighted FSE images. Column 2, needle tip void is visualized in axial, T1-weighted FSE images. Column 3, marker void is visualized on axial, T2*-weighted gradient-echo images. Note that there is minimal tissue motion throughout each procedure.

placement procedures and 3 biopsy procedures were performed. The average patient weight and height were $88 \mathrm{~kg}$ and $173 \mathrm{~cm}$ while the maximum weight and height were 132 $\mathrm{kg}$ and $183 \mathrm{~cm}$. Average patient age was 69 years (range 57 to 80 ). No patients had undergone prior radiation therapy, prostatectomy, hormone suppression therapy or transurethral prostate resection.

Average total duration for the MR procedure was 76 minutes (maximum 93, minimum 60), and the average time required for each of the 4 fiducial marker placements was 13 minutes including time for target selection, needle placement, fiducial marker deposition and all confirmatory imaging. Before each procedure an additional 15 minutes was required for administration of local anesthesia and 15 minutes for patient positioning.

The major patient complaint during the procedure was shoulder discomfort due to hyperextension of the joint when lying prone on the scanner table (this was relieved in later studies by using extra foam supports for the chest, which reduce the shoulder angle). Following the intervention, patients were observed for an average of 2 hours until successfully voiding without difficulty. During the 1 week followup 2 patients experienced hematuria - 1 had a single episode and the other had hematuria of 2 days duration, which then resolved spontaneously. One patient reported a single episode of hematochezia. None of the patients experienced rectal pain, urinary retention, infection, or other adverse events. All patients were free of symptoms at the end of the 1 week followup.

Intra-procedure imaging. In figure 5, column 1, typical T2-weighted fast spin-echo images acquired for target selection are shown. To improve visualization in and around the prostate, all images were intensity corrected, producing uniform signal images, as described previously. ${ }^{12}$ With the needle in place, the prostate was imaged again using a T1weighted fast spin-echo sequence, allowing for confirmation of needle placement accuracy (fig. 5, column 2). After each gold fiducial marker was deposited, T2*-weighted axial images were acquired to confirm marker location (fig. 5, column $3)$.

Needle and fiducial marker placement accuracy. Needle and fiducial marker placement accuracy were measured using the axial images acquired during the procedure. Needle accuracy was the absolute distance between the target location and the center of the needle tip bloom (measured using the 3 dimensional coordinates from the MR image volume). Fiducial marker placement accuracy was the absolute distance between the target point and the center of the marker void. The mean needle placement error was $1.9 \mathrm{~mm}$ (range 0.3 to 3.6 , fig. 6, $A$ ). The mean fiducial marker placement error was $4.8 \mathrm{~mm}$ (range 1.9 to 8.3 , fig. $6, B$ ). The mean fiducial marker placement error transverse to the needle 
direction was $2.6 \mathrm{~mm}$ (range 1.4 to 4.6 , fig. $6, C$ ). Because tissue biopsy cores are typically $1.5 \mathrm{~cm}$ long, transverse error measurements are more relevant for assessing the accuracy of the system for collecting tissue samples.

Biopsy needle placement accuracy. Three MR guided prostate biopsy procedures were performed. Biopsy locations were selected throughout the peripheral zone of the prostate using T2-weighted fast spin-echo images (fig. 7, A). Subsequently, after inserting the biopsy needle but before collecting the tissue core biopsy, T1-weighted fast spin-echo images were acquired to confirm biopsy needle placement accuracy (fig. 7, $B$ and $C$ ). A total of 15 tissue biopsies were collected. Mean biopsy needle placement accuracy was 1.8 $\mathrm{mm}$ (range 0.4 to 4.0 ), which was similar to the accuracy obtained using the fiducial marker placement needles. Biopsy cores showing normal and cancerous prostate tissue were collected and, as all patients were already diagnosed with prostate cancer, were used for studies investigating the molecular effects of radiation therapy.

\section{DISCUSSION}

Tissue targeting accuracy was measured by comparing the location of the gold fiducial markers, deposited in the tissue, after the needle had been removed. Assuming that the tissue returns to its original shape after the needle is withdrawn, the location of the deposited fiducial marker relative to the target point gives an accurate measure of tissue targeting accuracy. This assumption was confirmed by comparing images of the prostate acquired before and following needle placements (fig. 8).

As expected, the prostate was elastically deformed while the needle was within the tissue. The majority of markers were deposited in the tissue proximal to the target location (as measured along the needle insertion axis). As described, the needle and cannula were inserted $5 \mathrm{~mm}$ past the target point ( $8 \mathrm{~mm}$ at the prostate base) in the last 2 procedures in an effort to minimize the impact of tissue deformation. This technique notably improved fiducial marker placement accuracy, and average marker placement accuracy for the 4 procedures was 5.9, 5.1, 4.7 and $3.5 \mathrm{~mm}$, respectively. Improvements in patient positioning, which helped to immobilize the prostate, also contributed to this accuracy improvement. In particular, downward pressure was applied to the device before locking it into place, which immobilized the prostate against the pubic symphysis.

An alternative explanation for the fiducial marker placement errors is that the markers were not accurately deposited at the tip of the cannula or that they were suctioned back as the cannula and stylus were withdrawn from the tissue. Both prior experience with this deposition technique ${ }^{9}$ and experiments performed in clear polyacrylamide gel phantoms (results not shown) do not support this explanation.

As we are ultimately interested in the accuracy with which tissue samples can be acquired from the prostate, the transverse component of fiducial marker placement error was also examined (ie perpendicular to the needle insertion axis, fig. 6, C). Commonly, core tissue biopsies are $15 \mathrm{~mm}$ long and approximately $1.5 \mathrm{~mm}$ in diameter. Therefore, because the tissue core is so long, small errors in the
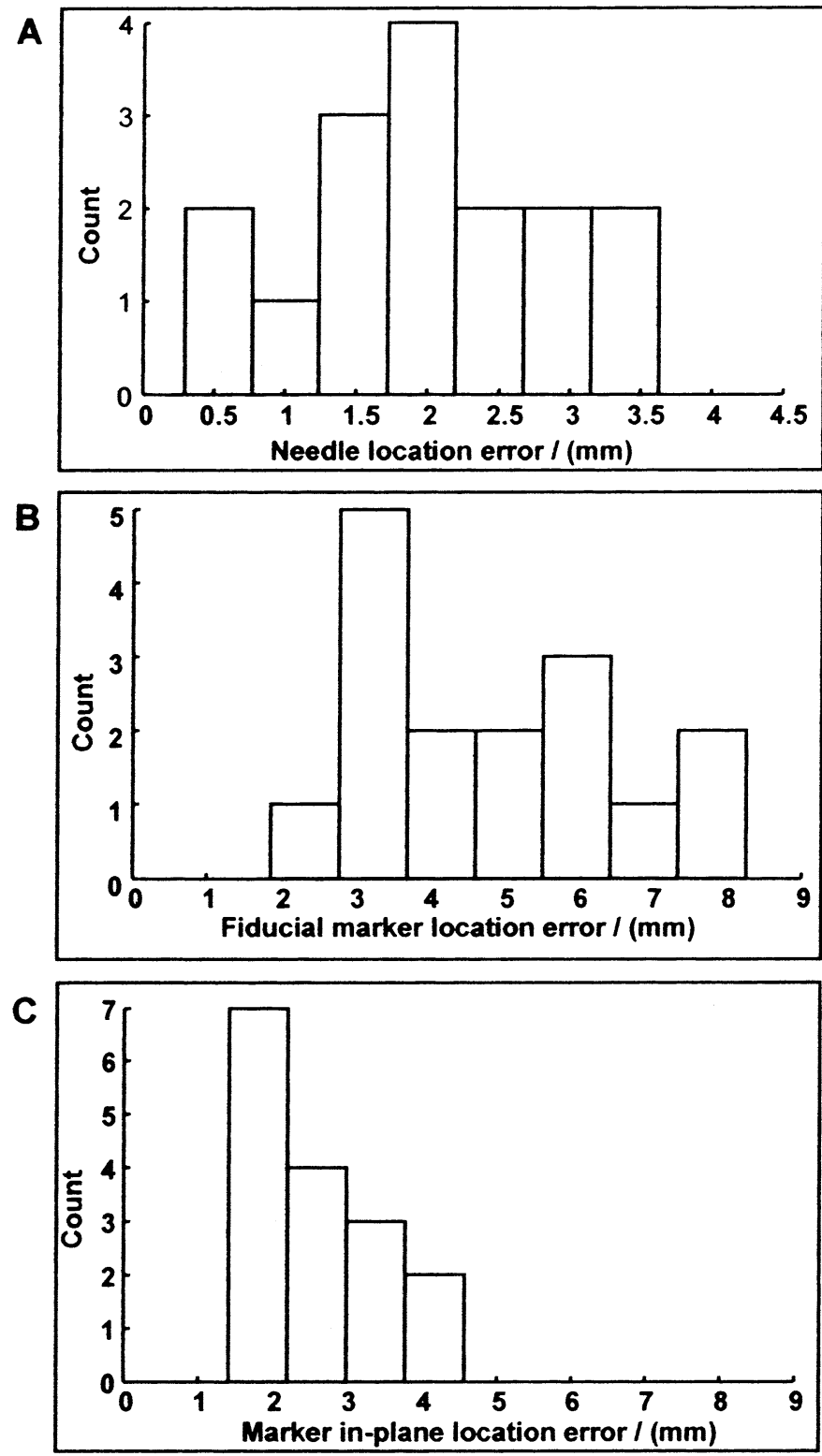

Fig. 6. Needle and fiducial-marker placement accuracy shown by error histograms for all 16 gold fiducial markers placed. $A$, needle tip location errors. $B$, fiducial marker location errors. $C$, fiducial marker in-plane location errors. Mean placement errors for each are $1.9,4.8$ and $2.6 \mathrm{~mm}$, respectively. Because tissue core biopsies are typically $1.5 \mathrm{~cm}$ long, last measure, fiducial marker in-plane placement error, is best predictor of tissue biopsy acquisition accuracy.

insertion depth of the biopsy needle are not of great consequence.

As all of our patients were already diagnosed with prostate cancer, biopsy cores were processed for studies examining the molecular effects of radiation therapy. While the present study has allowed us to establish the accuracy of MR guided needle placement and tissue targeting accuracy, we cannot yet comment on the overall, histologically validated sensitivity and specificity of MR guided diagnostic biopsy using this device. Subsequent studies with larger numbers of patients will be required to answer this question and must deal with confounding factors such as patient tumor size, patient selection (ie only patients in whom TRUS guided 

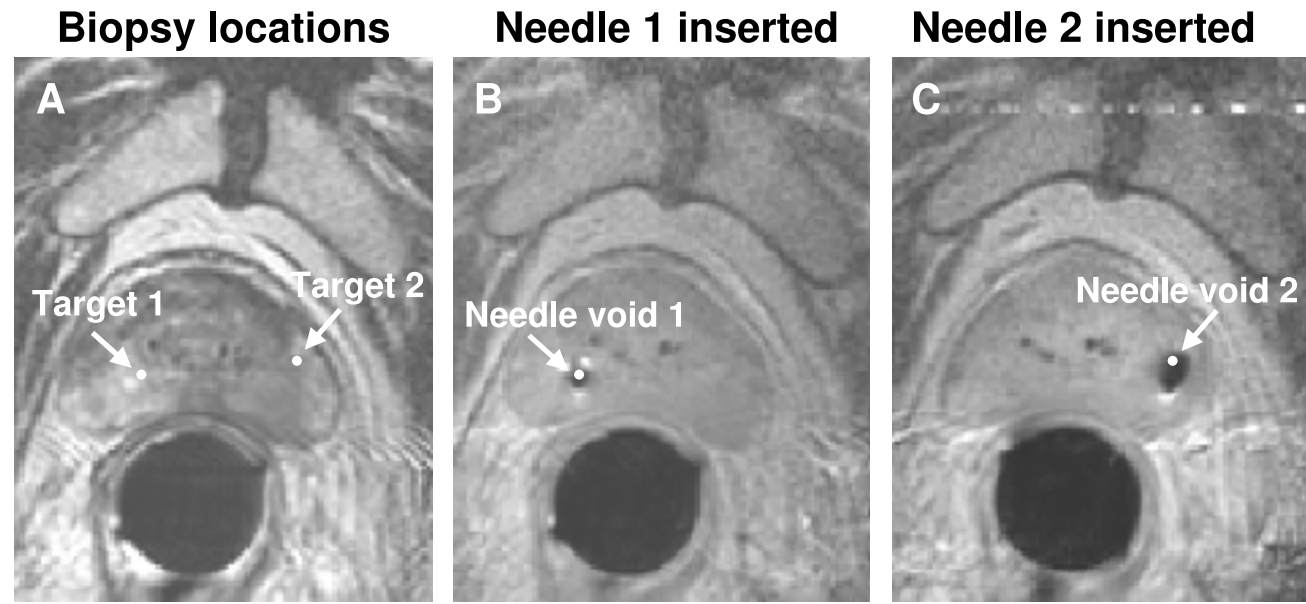

FIG. 7. Biopsy target and needle visualization images. $A$, biopsy locations were selected on axial, T2-weighted fast spin-echo images. $B$ and $C, 14$ gauge biopsy needle void is visualized in axial, T1-weighted fast spin-echo images.

prostate biopsy failed would be likely to advance to MR guided biopsy), and the sensitivity/specificity of the MR imaging sequences used. Moreover, as this technique is much more expensive and time consuming than TRUS guided prostate biopsy, its clinical usefulness remains to be established. Nevertheless, the application of this device for investigational image guided interventions can be foreseen.

\section{CONCLUSIONS}

We emphasized 4 key features of the system investigated in this study. First, the entire device is compact and, therefore, can be used in a standard $1.5 \mathrm{~T}$ cylindrical scanner architecture, even with larger patients (the largest patient treated was $132 \mathrm{~kg}, 4 \mathrm{~kg}$ less than the manufacturer limit for the scanner table). As standard scanner architectures are much more widely available than open models, this system could be applicable at many centers and moreover, can immediately take advantage of mainstream MRI hardware and pulse sequence development (such as the introduction of 3.0T whole body scanners). Second, the needle placement procedure is uncomplicated in that 4 targeted needle placements can be performed with an overall MR procedure time of approximately 1 hour (including time for confirmatory imaging after every needle and fiducial marker placement). Third, the endorectal sheath, which includes the local imag- ing coil and entirely contains the needle guide, remains stationary throughout the procedure. This design helps to prevent deformation of the prostate and surrounding tissues during the procedure, maintaining accurate registration of all image data sets and reducing the need for deformable registration techniques. ${ }^{13}$ Also, it prevents distention of the rectal wall during needle guide positioning, improving patient comfort. Finally, we do not rely heavily on real time imaging techniques. Other than the device tracking pulse sequence, no specialized pulse sequences are required when using this device. Therefore, we maintain the flexibility to use virtually any imaging technique, including MR spectroscopy and dynamic contrast enhancement, both of which have shown promise in the diagnosis and delineation of tumors within the prostate. Currently, we have focused on anatomical imaging techniques. Subsequent work will investigate the application of the device with pulse sequences that provide information about the functional and metabolic state of the tissue.

\section{ACKNOWLEDGEMENT}

Sharon Smith provided administrative support, Scott Chesnick advised on imaging and tracking coil design, Elliot McVeigh and Jeff Duyn provided scanner resources, Anton
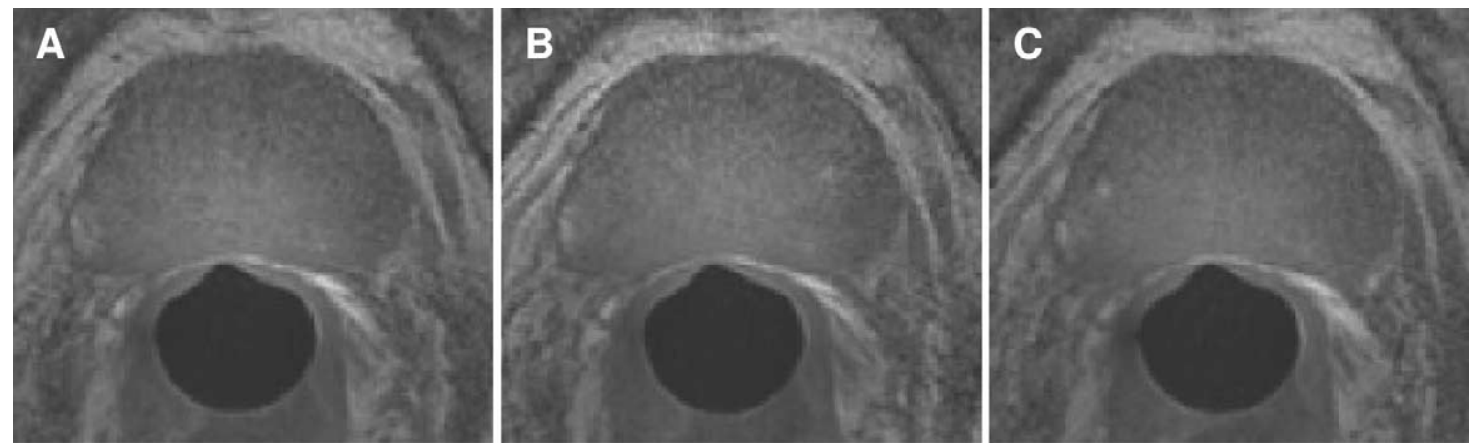

FiG. 8. T1-weighted axial fast-spin-echo images of prostate. $A$, acquired 20 minutes after start of procedure. $B, 40$ minutes after start of procedure. $C, 50$ minutes after start of procedure. Throughout intervention, including 4 needle placements, prostate and surrounding tissues remain stable. Importantly stationary endorectal sheath prevents movement of needle guide from causing tissue deformation. 
Deguet and Anand Viswanathan provided support software, and Karen Ullman assisted in developing the patient positioning system.

$\begin{aligned} & \text { Abbreviations and Acronyms } \\ \text { BW } & =\text { bandwidth } \\ \text { ETL } & =\text { echo train length } \\ \text { FA } & =\text { flip angle } \\ \text { FOV } & =\text { field of view } \\ \text { FSE } & =\text { fast spin echo } \\ \text { GE } & =\text { gradient echo } \\ \text { MRI } & =\text { magnetic resonance imaging } \\ \text { NEX } & =\text { number of excitations } \\ \text { T1 } & =\text { longitudinal relaxation time } \\ \text { T2 } & =\text { transverse relaxation time } \\ \text { TE } & \text { echo time } \\ \text { TR } & =\text { repetition time } \\ \text { TRUS } & \text { transrectal ultrasound }\end{aligned}$

\section{REFERENCES}

1. Yu, K. K. and Hricak, H.: Imaging prostate cancer. Radiol Clin North Am, 38: 59, 2000

2. Wefer, A. E., Hricak, H., Vigneron, D. B., Coakley, F. V., Lu, Y., Wefer, J. et al: Sextant localization of prostate cancer: comparison of sextant biopsy, magnetic resonance imaging and magnetic resonance spectroscopic imaging with step section histology. J Urol, 164: 400, 2000

3. Cormack, R. A., Kooy, H., Tempany, C. M. and D'Amico, A. V.: A clinical method for real-time dosimetric guidance of transperineal $125 \mathrm{I}$ prostate implants using interventional magnetic resonance imaging. Int J Radiat Oncol Biol Phys, 46: 207,2000

4. D’Amico, A. V., Tempany, C. M., Cormack, R., Hata, N., Jinzaki, M., Tuncali, K. et al: Transperineal magnetic resonance image guided prostate biopsy. J Urol, 164: 385, 2000
5. Cormack, R. A., D’Amico, A. V., Hata, N., Silverman, S., Weinstein, M. and Tempany, C. M.: Feasibility of transperineal prostate biopsy under interventional magnetic resonance guidance. Urology, 56: 663, 2000

6. Fei, B., Duerk, J. L., Boll, D. T., Lewin, J. S. and Wilson, D. L.: Slice-to-volume registration and its potential application to interventional MRI-guided radio-frequency thermal ablation of prostate cancer. IEEE Trans Med Imaging, 22: 515,2003

7. DiBiase, S. J., Hosseinzadeh, K., Gullapalli, R. P., Jacobs, S. C., Naslund, M. J., Sklar, G. N. et al: Magnetic resonance spectroscopic imaging-guided brachytherapy for localized prostate cancer. Int J Radiat Oncol Biol Phys, 52: 429, 2002

8. Kaplan, I., Oldenburg, N. E., Meskell, P., Blake, M., Church, P. and Holupka, E. J.: Real time MRI-ultrasound image guided stereotactic prostate biopsy. Magn Reson Imaging, 20: 295, 2002

9. Susil, R. C., Krieger, A., Derbyshire, J. A., Tanacs, A., Whitcomb, L. L., Fichtinger, G. et al: System for MR image-guided prostate interventions: canine study. Radiology, 228: 886 , 2003

10. Krieger, A., Susil, R. C., Menard, C., Coleman, J. A., Fichtinger, G., Atalar, E. et al: Design of a novel MRI compatible manipulator for image guided prostate interventions. IEEE Trans Biomed Eng, 52: 306, 2005

11. Parker, C. C., Damyanovich, A., Haycocks, T., Haider, M., Bayley, A. and Catton, C. N.: Magnetic resonance imaging in the radiation treatment planning of localized prostate cancer using intra-prostatic fiducial markers for computed tomography co-registration. Radiother Oncol, 66: 217, 2003

12. Susil, R. C., Camphausen, K., Choyke, P., McVeigh, E. R., Gustafson, G. S., Ning, H. et al: System for prostate brachytherapy and biopsy in a standard 1.5 T MRI scanner. Magn Reson Med, 52: 683, 2004

13. Mizowaki, T., Cohen, G. N., Fung, A. Y. and Zaider, M.: Towards integrating functional imaging in the treatment of prostate cancer with radiation: the registration of the MR spectroscopy imaging to ultrasound/CT images and its implementation in treatment planning. Int $J$ Radiat Oncol Biol Phys, 54: 1558,2002 